\title{
GABRG2 C588T gene polymorphisms might be a predictive genetic marker of febrile seizures and generalized recurrent seizures: a case-control study in a Romanian pediatric population
}

\author{
Anamaria Todoran Butilă ${ }^{1}$, Ancuta Zazgyva², Anca lleana Sin², Elisabeta Racoș Szabo ${ }^{3}$, \\ Mariana Cornelia Tilinca ${ }^{2}$
}

\author{
${ }^{1}$ Department of Genetics, University of Medicine and Pharmacy of Târgu-Mureş, \\ Târgu-Mureş, Romania \\ 2Department of Cell and Molecular Biology, University of Medicine and Pharmacy \\ of Târgu-Mureş, Târgu-Mureş, Romania \\ ${ }^{3}$ Department of Psychiatry, University of Medicine and Pharmacy of Târgu-Mureş, \\ Târgu-Mureş, Romania
}

Submitted: 10 June 2016

Accepted: 14 August 2016

Arch Med Sci 2018; 14, 1: 157-166

DOI: 10.5114 /aoms.2016.63739

Copyright (C) 2016 Termedia \& Banach

\author{
Corresponding author: \\ Ancuta Zazgyva MD, PhD \\ Department of \\ Cell and Molecular Biology \\ University of Medicine \\ and Pharmacy \\ of Târgu-Mureş \\ 38 Gheorghe Marinescu St \\ 540139 Târgu-Mureş \\ Romania \\ Phone: +40745612 397 \\ E-mail: zazgyvaa@yahoo.com
}

\begin{abstract}
Introduction: This case-control study aimed to assess two single nucleotide polymorphisms of the gene encoding the GABRG2 protein - GABRG2 (3145 G>A) and GABRG2 rs 211037 Asn196Asn (C588T) - in a cohort of pediatric patients from Romania, and evaluate their possible impact on drug-resistant forms of generalized epilepsy and recurrent febrile seizures.

Material and methods: One hundred and fourteen children with idiopathic generalized epilepsy (group 1) or febrile seizures (group 2) were compared to 153 controls. Peripheral blood samples were assessed using polymerase chain reaction-restriction fragment length polymorphism analysis, with results interpreted based on the disappearance of a restriction site in the C allele (122 bp) compared to the T allele (100 bp $+22 \mathrm{bp}$ ).

Results: A significant association was found with the TT homozygous genotype and T allele for both febrile seizures and epilepsy for the C588T locus, while GABRG2 G>A 3145 showed no significant association with any type of seizure. The TT homozygous genotype of GABRG2 Asn196Asn polymorphism was more frequent in patients with a history of febrile seizures ( $p=$ 0.0001 ), without a significant association identified for GABRG2-G>A 3145. Composite analysis showed associations with epilepsy for CC-AG $(p=0.02)$ and CT-AG $(p=0.007)$ with the CC-AA combination as reference.

Conclusions: C588T polymorphism of the GABRG2 gene might be a predictive genetic marker in triggering febrile convulsions. GABRG2 rs211037 TT homozygotes and $T$ allele variants have an increased risk for developing febrile seizures. Recurrent crises and repeated episodes of seizures are more frequent in the GABRG2 Asn196Asn TT genotype polymorphism, with a 45 and 8 times higher risk of developing idiopathic generalized epilepsy and recurrent febrile seizures, respectively.
\end{abstract}

Key words: idiopathic epilepsy, $\mathrm{GABA}_{\mathrm{A}}$ receptors, febrile convulsions. 


\section{Introduction}

In recent years, genetic research has been widely used in many medical specialties, in both the diagnosis and risk stratification algorithms of diverse pediatric and adult diseases [1-4]. In terms of neurological pathology in children, hereditary disorders are relatively frequent in the pediatric population, and more common than those found in adults [5].

Epilepsy is a condition that affects an estimated 10.5 million children worldwide, and idiopathic generalized epilepsies (IGE) represent around 1/3 of all epilepsies. There is increasing evidence that genetic factors play an important role in the development of idiopathic epilepsy; this is mostly based on epidemiological studies, including those of familial aggregation, monozygotic twins and families with a history of epilepsy [6]. Of these, IGE seems to have the most significant hereditary component [6-8]. In the last decade an increasing number of reports have appeared on rare forms of idiopathic epilepsy with proven Mendelian inheritance [9]. These types of epilepsies are characterized by recurrent generalized seizures with childhood- or adolescence-onset, usually in patients with normal development, and no neurological impairment or structural lesions detectable by imaging [10]. The syndromes include juvenile myoclonic epilepsy, childhood absence epilepsy, juvenile absence epilepsy, and primary generalized tonic-clonic seizures. The seizures have specific clinical characteristics in each individual syndrome, and typical electroencephalographic (EEG) epileptiform discharges.

Gamma-aminobutyric acid (GABA) is the major inhibitory neurotransmitter in the adult brain, acting through two classes of specific receptors: $\mathrm{GABA}_{\mathrm{A}}$ and $\mathrm{GABA}_{\mathrm{B}}$; these are molecular targets for anticonvulsant drugs, either through a direct action or an increase in GABA levels [11]. The $G A B A_{A}$ receptor is the most common type of receptor found in the brain, with specific binding sites for benzodiazepines and barbiturates [12, 13]. Functional changes of $G_{A B A}$ and $G_{A B A}$ receptors have been identified in both human epilepsy and animal models of the disease [14-16]. Genes encoding the $\alpha 1, \beta 2$ and $\gamma 2$ subunits of the receptor (GABRA1, GABRB2 and GABRG2) might have a role in the onset of IGE because of the extended distribution of these receptors throughout the central nervous system, the possibility to produce postsynaptic inhibition, and the potential modulation of common antiepileptic drugs' action [12]. Various reports claim an association of mutations in the genes encoding $\mathrm{GABA}_{\mathrm{A}}$ receptors with monogenic forms of IGE $[10,17]$. To date, epilepsy-causing mutations have been identified in subunits $\alpha 1, \gamma 2, \beta 3$ and $\delta$ [18]. These studies suggest that functional loss of the genes encoding the $G_{A B A}$ receptor is an important mechanism in the pathogenesis of epileptic syndromes that have genetic determinism, and although many other mechanisms are involved in the pathogenesis of IGE, these findings provide important insights into the development of these "idiopathic epilepsies" [10]. Recently identified mutations in the $\gamma 2$ subunit of the GABA receptor, with the encoding gene located on the long arm of chromosome 5 (5q34), were reported in two families: in the first case the phenotype described was compatible with generalized epilepsy with febrile seizures plus (GEFS+), while in the second case the phenotype was characteristic for childhood absence epilepsy with febrile seizures plus $[19,20]$. Also several studies have found the association of mutations in this gene with IGE and febrile seizures (FS) [21].

The main objective of this study was to assess two single nucleotide polymorphisms of the gene encoding the GABRG2 protein - GABRG2 (3145 G>A) and GABRG2 rs 211037 Asn196Asn (C588T) - in a cohort of pediatric patients from Romania, based on the hypothesis that these polymorphisms are potential predictive markers in generalized convulsive seizures and FS. The possible impacts of these polymorphisms on drug-resistant forms of generalized epilepsy and recurrent FS were evaluated as secondary objectives.

\section{Material and methods}

The study had a case-control design, and was approved by the local Ethics Committee. All legal caregivers signed an informed consent form for participating in the study.

\section{Patient selection}

The study included a cohort of 114 children hospitalized in a local Clinic of Pediatric Neurology and Psychiatry, or treated in the wards and outpatient centers of the Pediatric Neuropsychiatry Service between May 2011 and October 2013. These cases were divided into two groups: patients with IGE (group 1, 60 patients aged 2-17 years) and patients with FS (group 2, 54 children aged 1.5-5 years).

Epilepsy was confirmed by a history of at least 2 unprovoked seizures (except FS, single episodes of seizures and seizures occurring in the neonatal period), accompanied by specific EEG epileptiform changes, in a patient with normal neurological development and normal neurological examination, and no structural lesions detectable by imaging. Patients with an uncertain diagnosis, a single episode of seizure, provoked seizures, secondary epilepsies or psychiatric disorders were excluded from the study; noncompliant patients were also excluded. 


\section{Subgroup with generalized recurrent seizures}

Patients were divided based on the response to antiepileptic medication in order to identify predictive factors for developing recurrent-type epileptic seizures or repeated FS. The definition of drug resistance was based on the recommendations of the International League Against Epilepsy [22] - no control of seizures during a year of well-directed treatment in appropriate doses and combinations.

\section{Control group}

The control group consisted of 153 children aged 2-17 years, hospitalized in the Clinic of Pediatric Neurology and Psychiatry or other pediatric services with no neurological disorders, mental illness, or personal/family history of seizures.

\section{Molecular analysis}

Peripheral blood samples $(2 \mathrm{ml})$ collected from both cases and controls were kept at $4^{\circ} \mathrm{C}$ until DNA extraction, and stored at $-20^{\circ} \mathrm{C}$ afterwards, before processing. For polymerase chain reaction-restriction fragment length polymorphism (PCR-RFLP) analysis, DNA was isolated using the Zymo Beads Genomic DNA extraction commercial kit (Zymo Research) according to the manufacturer's instructions, followed by DNA amplification by PCR and fragmentation by enzymatic digestion using specific restriction enzymes. Digestion products were stained with ethidium bromide (Promega, USA), followed by gel electrophoresis in $2 \%$ agarose in a UV transillumination system coupled with a camera for image capture (Vilber Lourmat Imaging System). Table I presents the primer sequences needed for amplification, specific restriction enzymes and fragment lengths.

The protocol was based on the methodology described by Chou et al. [16], with a few adaptations: initial denaturation for $5 \mathrm{~min}$ at $95^{\circ} \mathrm{C}$ was followed by 37 cycles (for GABGRG2 $3145 \mathrm{G}>\mathrm{A}$ ) or 35 cycles (for GABGRG2 Asn196Asn) of denaturation for $30^{\prime \prime}$ at $94^{\circ} \mathrm{C}$, attachment of primers (annealing) for $30^{\prime \prime}$ at $60^{\circ} \mathrm{C}$ for GABGRG2 3145 $\mathrm{G}>\mathrm{A}$ and $55^{\circ} \mathrm{C}$ for GABGRG2 Asn196Asn, then extension for $45^{\prime \prime}$ at $72^{\circ} \mathrm{C}$, with a final extension for $7^{\prime}$ at $72^{\circ} \mathrm{C}$ in both cases. As C to T substitution at nucleotide position 588 in exon 5 of the GABRG2 gene creates a restriction site for Apol (the restriction enzyme), results were interpreted based on the disappearance of a restriction site in the $\mathrm{C}$ allele (122 bp) compared to the $\mathrm{T}$ allele (100 bp +22 bp), shown schematically in Figure $1 \mathrm{~A}$ (the fragment with $<100 \mathrm{bp}$ is not visible after electrophoresis). Figure $1 \mathrm{~B}$ presents the genotypes for GABRG2 locus C588T, after amplification, enzymatic digestion and electrophoresis.

\section{Statistical analysis}

Statistical analysis was performed using Statistical Package for Social Sciences (SPSS, version 17,

Table I. Sequences of primers, restriction enzyme and length of obtained fragments

\begin{tabular}{|lccc|}
\hline SNP & \multicolumn{1}{c}{ Sequencing primer } & Fragments & $\begin{array}{c}\text { Restriction } \\
\text { enzyme }\end{array}$ \\
\hline GABGRG2 & FW: 5'-AGA AAT TTA CCA ACT GGT CTA GCC GG-3' & AA & Ncil \\
$(3145$ G $>$ A $)$ & Rev: 5'-AAA TCA AAT ATT GTG TCA TGC TTA GT -3' & AG & \\
& & GG & Apol \\
\hline GABGRG2 & FW: 5'-GAG TGC CAA TTA CAA TTG CAA AA-3' & CC: 122 bp & \\
$(211037)$ & Rev: 5'-AAT CAG AAA GAC TGT AGG TGA GG-3' & CT: 122 bp + 100 bp + 22 bp; & \\
Asn196Asn & & TT: $100+22$ bp & \\
C588T $)$ & & & \\
\hline
\end{tabular}

A

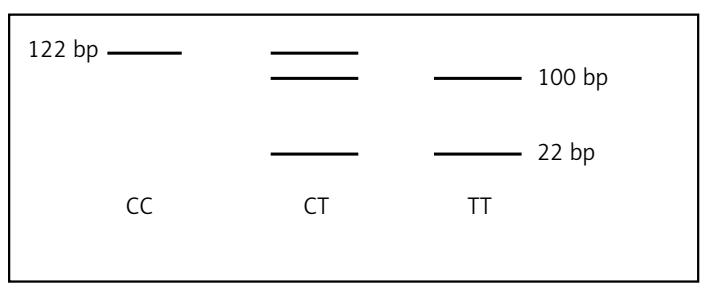

\section{B}

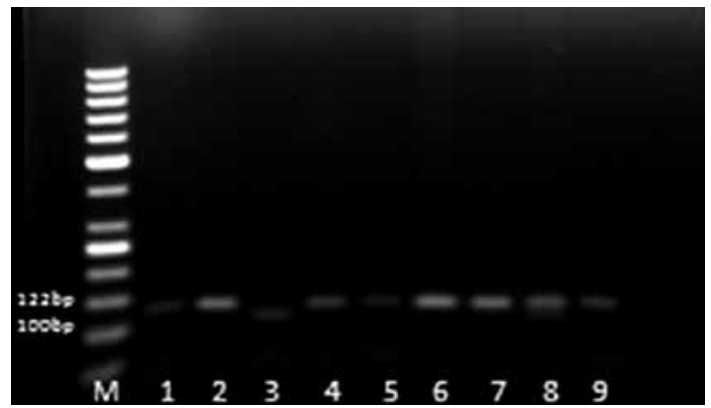

Figure 1. A - Fragment lengths after enzymatic digestion (GABRG2 C588T); B - genotypes for GABRG2 locus C588T, after amplification, enzymatic digestion (with Apol) and electrophoresis in agarose gel $M-$ marker; 1, 2, 4, 5, 6, 7, 9-CC, 8-CT, 3-TT. 
Chicago, IL, USA), with data considered as normal or quantitative variables. Frequencies were used for normal variables, while quantitative variables were tested for normal distribution using the Kolmogorov-Smirnov test, and characterized by median and percentages (25-75\%) or mean and standard deviation (SD) when appropriate. The $\chi^{2}$ test was used for comparing more than 2 variables and calculating the Hardy-Weinberg equilibrium. The odds ratio (OR) and 95\% confidence interval $(\mathrm{Cl})$ were calculated to demonstrate the probability or susceptibility of the association of gene polymorphisms with treatment-resistant epilepsies. Statistical significance was set at $p<0.05$. Quantitative variables were compared using the Mann-Whitney test [23].

\section{A}

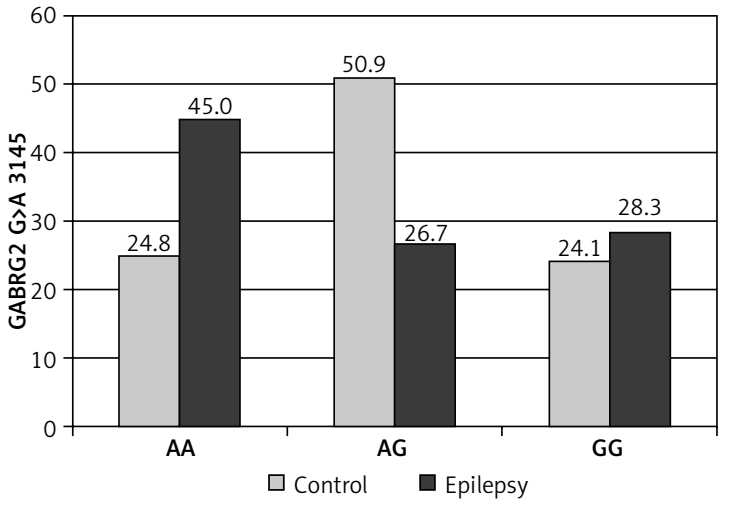

C

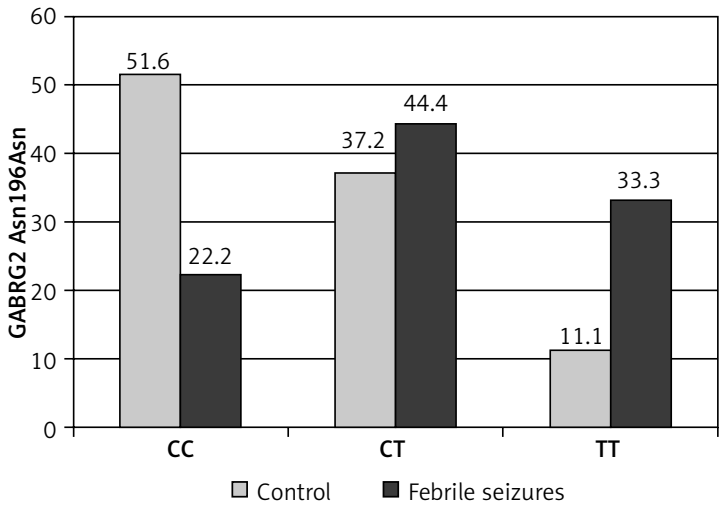

\section{Results}

Assessing the frequency of GABRG2 polymorphisms in children with epilepsy

Distribution of GABRG2 rs211037 and GABRG2 G>A in nucleotide position 3145 in all groups met Hardy-Weinberg equilibrium conditions. The frequencies for the polymorphisms of these mutations were determined for the first time in a population from Romania, and are presented in Figure 2. Tables II and III present allele frequency and genotype distribution of GABRG2 C588T in groups 1 and 2 - patients with the $T$ allele and homozygous for the TT variant were strongly predisposed to developing FS when compared to those with a CC genotype.

\section{B}

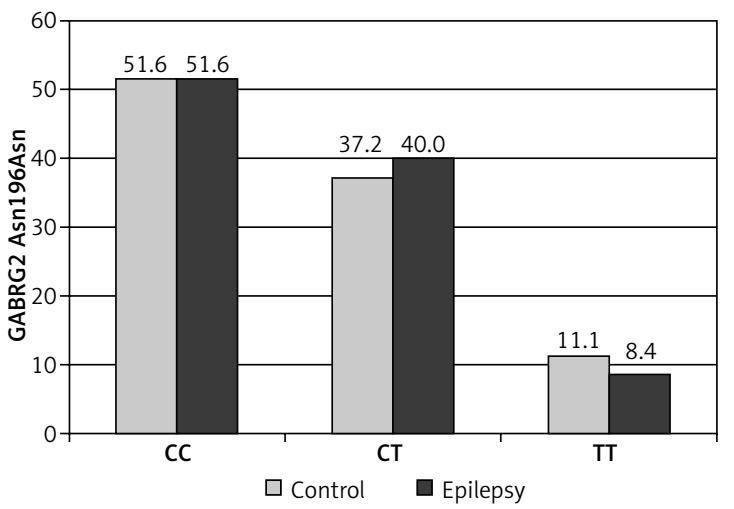

Figure 2. A - Genotype frequency for the GABRG2 G>A polymorphism in IGE, B - genotype frequency for the GABRG2 Asn196Asn polymorphism in IGE, C - genotype frequency for the GABRG2 Asn196Asn polymorphism in FS

Table II. Allele frequency and genotype distribution of GABRG2 C588T in the epilepsy, febrile seizures and control groups

\begin{tabular}{|lcc|}
\hline $\begin{array}{l}\text { Genotype } \\
\text { GABRG2 C588T }\end{array}$ & $\begin{array}{c}\text { Generalized epilepsy vs. control } \\
\chi^{2} p \text {-value, OR, 95\% Cl }\end{array}$ & $\begin{array}{c}\text { Febrile seizures vs. control } \\
\chi^{2} p \text {-value; OR, 95\% Cl }\end{array}$ \\
\hline CC vs. CT & $p=0.82, \mathrm{OR}=1.07,0.57-2.02$ & $p=0.008^{*}, \mathrm{OR}=2.77,1.28-6.001$ \\
\hline CC vs. TT & $p=0.60, \mathrm{OR}=0.74,0.25-2.20$ & $p<0.0001^{*}, \mathrm{OR}=6.97,2.84-17.13$ \\
\hline C vs. T & $p=0.77, \mathrm{OR}=0.93,0.58-1.49$ & $p<0.0001^{*}, \mathrm{OR}=2.95,1.87-4.64$ \\
\hline CC vs. CT + TT & $p=0.001^{\star}, \mathrm{OR}=0.26,0.11-0.60$ & $p=0.0002^{*}, \mathrm{OR}=0.26,0.13-0.54$ \\
\hline TT vs. CT + CC & $p=0.0009^{*}, \mathrm{OR}=5.5,1.87-16.1$ & $p=0.0002^{*}, \mathrm{OR}=4.00,1.87-8.53$ \\
\hline
\end{tabular}

*Statistically significant, $\mathrm{OR}$ - odds ratio, $\mathrm{Cl}$ - confidence interval. 
Table III. Alleles and genotype distribution for GABRG2 in patients from group 1 and controls, and genotype and allele frequency in patients with recurrent seizures

\begin{tabular}{|c|c|c|c|}
\hline Genotype 3145 GABRG2 & $\begin{array}{l}\text { Group } 1 \\
N(\%)\end{array}$ & $\begin{array}{c}\text { Control group } \\
N(\%)\end{array}$ & $\chi^{2}$ test $p$-value, OR, $95 \% \mathrm{Cl}$ \\
\hline AA & $27(45.0)$ & $38(24.8)$ & - \\
\hline$A G$ & $16(26.7)$ & $78(50.9)$ & $p=0.006^{\star}, \mathrm{OR}=0.28,0.13-0.59$ \\
\hline GG & $17(28.3)$ & $37(24.1)$ & $p=0.28, \mathrm{OR}=0.64,0.30-1.32$ \\
\hline A & $70(58.3)$ & $154(50.3)$ & - \\
\hline G & $50(41.7)$ & $152(49.7)$ & $p=0.13, \mathrm{OR}=0.72,0.47-1.11$ \\
\hline$G G$ vs. $A G+A A$ & $17 / 43$ & $37 / 116$ & $p=0.53, \mathrm{OR}=1.24,0.63-2.43$ \\
\hline$A A$ vs. $A G+G G$ & $27 / 33$ & $38 / 115$ & $p=0.004^{\star}, \mathrm{OR}=0.40,0.21-0.75$ \\
\hline Genotype frequency & $\begin{array}{c}\text { Drug-resistant } \\
\text { forms of idiopathic } \\
\text { generalized epilepsy } \\
\begin{array}{c}N=11 \\
n(\%)\end{array}\end{array}$ & $\begin{array}{c}\text { Well-controlled } \\
\text { forms of idiopathic } \\
\text { generalized epilepsy } \\
\begin{array}{c}N=49 \\
n(\%)\end{array}\end{array}$ & $\chi^{2}$ test $p$-value, OR, $95 \% \mathrm{Cl}$ \\
\hline \multicolumn{4}{|l|}{ GABRG2 C588T: } \\
\hline $\mathrm{CC}$ & $1(9.1)$ & $30(61.2)$ & - \\
\hline $\mathrm{CT}$ & $7(63.6)$ & $17(34.7)$ & $p=0.02^{*}, \mathrm{OR}=12.35,1.39-109.13$ \\
\hline TT & $3(27.3)$ & $2(4.1)$ & $p=0.002^{*}, \mathrm{OR}=45.0,3.09-655.33$ \\
\hline C & $9(40.9)$ & $77(78.6)$ & - \\
\hline T & $13(59.1)$ & $21(21.4)$ & $p=0.001^{*}, \mathrm{OR}=5.29,1.99-14.07$ \\
\hline \multicolumn{4}{|l|}{ GABRG2 G>A 3145: } \\
\hline AA & $6(54.5)$ & $21(52.8)$ & - \\
\hline AG & $0(0.0)$ & $12(24.5)$ & - \\
\hline GG & $5(45.5)$ & $16(32.7)$ & $p=0.89, \mathrm{OR}=1.09,0.28-4.23$ \\
\hline A & $12(54.5)$ & $54(55.1)$ & - \\
\hline G & $10(45.5)$ & $44(44.9)$ & $p=0.96, \mathrm{OR}=1.02,0.40-2.59$ \\
\hline Genotype frequency & $\begin{array}{l}\text { Recurrent febrile } \\
\text { seizures } \\
N=16 \\
n(\%)\end{array}$ & $\begin{array}{l}\text { Single episode febrile } \\
\text { seizure } \\
N=38 \\
n(\%)\end{array}$ & $\chi^{2}$ test $p$-value, OR, $95 \% \mathrm{Cl}$ \\
\hline \multicolumn{4}{|l|}{ GABRG2 C588T: } \\
\hline CC & $2(12.5)$ & $10(26.3)$ & - \\
\hline CT & $3(18.7)$ & $22(57.9)$ & $p=0.85, \mathrm{OR}=0.45,0.05-3.70$ \\
\hline TT & $11(68.8)$ & $7(18.4)$ & $p=0.04^{\star}, \mathrm{OR}=7.85,1.31-47.06$ \\
\hline C & $7(21.9)$ & $42(53.8)$ & - \\
\hline $\mathrm{T}$ & $25(78.1)$ & $36(46.2)$ & $p=0.004^{*}, \mathrm{OR}=4.16,1.61-10.76$ \\
\hline
\end{tabular}

${ }^{*}$ Statistically significant, $\mathrm{OR}$ - odds ratio, $\mathrm{Cl}$ - confidence interval.

Assessing GABRG2 polymorphisms in cases of generalized recurrent seizures

According to their response to antiepileptic medication, $18.3 \%$ of patients with IGE had drug-resistant forms of epilepsy and $29.6 \%$ of patients with a history of FS had repeated episodes during febrile states triggered by respiratory tract infections (Table III). For those with recurrent seizures, a significant association was found with the TT homozygous genotype and T allele for both FS and epilepsy for the C588T locus, while GABRG2 G>A 3145 has not been identified as a factor associated with any type of seizure. 
Genotype distribution of the two gene polymorphisms in epileptic patients with a history of FS

When comparing patients in group 1 with and without a history of FS to identify a possible genotype-phenotype correlation, a statistically significant difference was found for the GABRG2 Asn196Asn polymorphism: the TT homozygous genotype was more frequent in patients with a history of FS ( $p=$ 0.0001 ). The same comparison showed no significant association for GABRG2-G>A 3145 (Table IV).

Genotype distribution of the two gene polymorphisms in epileptic patients by sex

For patients with IGE the GG genotype was more frequently found in girls (43.3\%) and the AA genotype was more frequent in boys (63.3\%). In the control group, the AA homozygous genotype was representative in girls (30\%), whereas the AG heterozygous genotype was dominant in boys $(69.8 \%)$, with a significant difference in both cases $(p=0.001)$. For the GABRG2 Asn 196 Asn variant, genotype distribution between the sexes showed no statistical significance (Table V).

Genotype-haplotype combinations (linked analysis of the two polymorphisms in the IGE group)

In order to identify an association linking the two polymorphisms with seizures, a composite analysis of several genotypes was performed. Of the nine possible variants, associations with epi-

Table IV. GABRG2 - Asn196Asn and GABRG2-G>A 3145 polymorphism in febrile seizures

\begin{tabular}{|c|c|c|c|c|c|c|c|c|c|}
\hline \multirow{2}{*}{\multicolumn{2}{|c|}{$p=0.0001$}} & \multicolumn{2}{|c|}{ Febrile seizures } & \multirow[t]{2}{*}{ Total } & \multirow{2}{*}{\multicolumn{2}{|c|}{$p=0.45$}} & \multicolumn{2}{|c|}{ Febrile seizures } & \multirow[t]{2}{*}{ Total } \\
\hline & & Yes & No & & & & Yes & No & \\
\hline \multicolumn{5}{|c|}{ GABRG2- Asn196Asn: } & \multicolumn{5}{|c|}{ GABRG2 G>A 3145: } \\
\hline CC & $N(\%)$ & $3(25.0)$ & $28(58.3)$ & $31(51.7)$ & AA & $N(\%)$ & $5(41.7)$ & $22(45.8)$ & $27(45.0)$ \\
\hline CT & $N(\%)$ & $5(41.7)$ & 19 (39.6) & $24(40.0)$ & AG & $N(\%)$ & $2(16.7)$ & $14(29.2)$ & $16(26.7)$ \\
\hline $\mathrm{TT}$ & $N(\%)$ & $4(33.3)$ & $1(2.1)$ & $5(8.3)$ & GG & $N(\%)$ & $5(41.7)$ & $12(25.0)$ & $17(28.3)$ \\
\hline Total & $N(\%)$ & $12(100.0)$ & $48(100.0)$ & $60(100.0)$ & Total & $N(\%)$ & $12(100.0)$ & $48(100.0)$ & $60(100.0)$ \\
\hline
\end{tabular}

Table V. GABRG2 G>A 3145 and GABRG2 Asn 196 Asn polymorphism by gender

\begin{tabular}{|c|c|c|c|c|c|c|}
\hline \multirow[t]{2}{*}{ Patient groups } & & & & \multicolumn{2}{|c|}{ Gender } & \multirow[t]{2}{*}{ Total } \\
\hline & & & & Female & Male & \\
\hline \multirow{4}{*}{$\begin{array}{l}\text { Cases (groups } 1 \text { and 2) } \\
p=0.001\end{array}$} & \multirow[t]{3}{*}{ GABRG2 INTRON } & $A A$ & $N(\%)$ & $8(26.7)$ & $19(63.3)$ & $27(45.0)$ \\
\hline & & $A G$ & $N(\%)$ & $9(30.0)$ & $7(23.3)$ & $16(26.7)$ \\
\hline & & GG & $N(\%)$ & $13(43.3)$ & $4(13.3)$ & $17(28.3)$ \\
\hline & Total & & $N(\%)$ & $30(100.0)$ & $30(100.0)$ & $60(100.0)$ \\
\hline \multirow{4}{*}{$\begin{array}{l}\text { Controls } \\
p=0.001\end{array}$} & \multirow[t]{3}{*}{ GABRG2 INTRON } & $A A$ & $N(\%)$ & $27(30.0)$ & $11(17.5)$ & $38(24.8)$ \\
\hline & & AG & $N(\%)$ & $34(37.8)$ & $44(69.8)$ & $78(51.0)$ \\
\hline & & GG & $N(\%)$ & $29(32.2)$ & $8(12.7)$ & $37(24.2)$ \\
\hline & Total & & $N(\%)$ & $90(100.0)$ & $63(100.0)$ & $153(100.0)$ \\
\hline \multirow{4}{*}{$\begin{array}{l}\text { Cases (groups } 1 \text { and 2) } \\
p=0.052\end{array}$} & \multirow[t]{3}{*}{ GABRG2 Asn 196 Asn } & $\mathrm{CC}$ & $N(\%)$ & $11(36.7)$ & $20(66.7)$ & $31(51.7)$ \\
\hline & & $\mathrm{CT}$ & $N(\%)$ & $15(50.0)$ & $9(30.0)$ & $24(40.0)$ \\
\hline & & TT & $N(\%)$ & $4(13.3)$ & $1(3.3)$ & $5(8.3)$ \\
\hline & Total & & $N(\%)$ & $30(100.0)$ & $30(100.0)$ & $60(100.0)$ \\
\hline \multirow{4}{*}{$\begin{array}{l}\text { Controls } \\
p=0.07\end{array}$} & \multirow[t]{3}{*}{ GABRG2 Asn 196 Asn } & $\mathrm{CC}$ & $N(\%)$ & $39(43.3)$ & $40(63.5)$ & $79(51.6)$ \\
\hline & & CT & $N(\%)$ & $38(42.2)$ & $19(30.2)$ & $57(37.3)$ \\
\hline & & $\mathrm{TT}$ & $N(\%)$ & $13(14.4)$ & $4(6.3)$ & $17(11.1)$ \\
\hline & Total & & $N(\%)$ & $90(100.0)$ & $63(100.0)$ & $153(100.0)$ \\
\hline
\end{tabular}


Table VI. Haplotypes in the control and epilepsy groups

\begin{tabular}{|c|c|c|c|c|c|}
\hline \multicolumn{3}{|c|}{ GABRG2 C588T-GABRG2 INTRON } & \multicolumn{2}{|c|}{ Patient groups } & \multirow[t]{2}{*}{$\chi^{2}$ test $p$-value, OR, $95 \% \mathrm{Cl}$} \\
\hline & & & Cases & Controls & \\
\hline \multirow[t]{9}{*}{ Haplotypes } & CC-AA & $N(\%)$ & $17(28.3)$ & $23(15.0)$ & Reference \\
\hline & CC-AG & $N(\%)$ & $11(18.3)$ & $43(28.1)$ & $p=0.02^{*}, \mathrm{OR}=0.34,0.13-0.86$ \\
\hline & CC-GG & $N(\%)$ & $3(5.0)$ & $13(8.5)$ & $p=0.09, \mathrm{OR}=0.31,0.07-1.27$ \\
\hline & CT-AA & $N(\%)$ & $10(16.7)$ & $8(5.3)$ & $p=0.35, \mathrm{OR}=1.69,0.55-5.19$ \\
\hline & CT-AG & $N(\%)$ & $5(8.3)$ & $30(19.6)$ & $p=0.007^{\star}, \mathrm{OR}=0.22,0.07-0.70$ \\
\hline & CT-GG & $N(\%)$ & $9(15.0)$ & $19(12.4)$ & $p=0.38, \mathrm{OR}=0.64,0.23-1.76$ \\
\hline & TT-AA & $N(\%)$ & 0 & $7(4.6)$ & - \\
\hline & TT-AG & $N(\%)$ & 0 & $5(3.2)$ & - \\
\hline & TT-GG & $N(\%)$ & $5(8.3)$ & $5(3.2)$ & $p=0.66, \mathrm{OR}=1.35,0.33-5.42$ \\
\hline Total & & $N(\%)$ & $60(100.0)$ & $153(100.0)$ & \\
\hline
\end{tabular}

lepsy were found for CC-AG $(p=0.02)$ and CT-AG $(p=0.007)$, using the CC-AA combination as a reference (Table $\mathrm{VI})$.

\section{Discussion}

Triggered by a rising fever without a proven central nervous system infection, FS are non-epileptic critical events occurring in children aged 6 months to 5 years, and represent the most common cause of seizures in childhood, with an incidence of $2-5 \%$ [6]. In spite of adequate treatment, FS may be recurrent in $30 \%$ of cases, with a $2-3 \%$ risk of developing subsequent epilepsy. Understanding the mechanism of recurrent seizures is paramount for their management. Although the etiology of FS is not clear, genetic factors seem to play a role in triggering them $[6,11,15]$. As the incidence of seizures in first degree relatives can reach $30 \%$, most authors consider FS a polygenic or multifactorial condition, with a possible autosomal dominant inheritance with incomplete penetrance [6, 8, 24-26].

The incidence of FS varies worldwide, with a lower incidence in Western Europe and the United States (2-5\%) compared to Asia (5-10\% in India, and $9 \%$ in Japan). Published evidence showed that the CC genotype for GABRG2 (rs211037) polymorphism in Asians with FS is frequent compared to controls; however, no such difference was identified in Caucasians. Thus it is possible that the CC genotype acts as a causal factor for the development of FS in Asians (Chinese and Egyptians), but not in Caucasians, perhaps due to differences in environmental factors, gene pool, or the linkage of this polymorphism with other variants with risk of epilepsy [27-30]. Nevertheless, further research is needed to clarify these aspects. A large num- ber of genes have been discussed as potentially involved in the hereditary transmission of FS [30]. From these, several mutations in the $\gamma 2$ subunit of the GABA receptor were reported to be associated with FS (2- (R43Q), 2 (K289M), 2 (Q351X), and 2 (IVS6 + 2 T -> G)) [19, 20, 31].

Through a comparative analysis of genotypes in patients with IGE and healthy patients, our results revealed that the GABRG2 polymorphism in the intronic position $3145 \mathrm{G}>\mathrm{A}$ is not associated with an increased risk of epilepsy. Based on published literature, the AA genotype was taken as a reference; for this genetic locus, GG and AG genotype frequency was $75 \%$ of the variants identified in the control group, while in the group of patients with epilepsy these genotypes were present in $55 \%$ of cases. Still, it is noteworthy that the A allele frequency was about $58.3 \%$ in the epilepsy group, close to that of the control group (50.3\%). As opposed to this, the AG heterozygous genotype was statistically significant in the epilepsy group $(p=0.006)$, with the same association noted in the dominant model ( $A A$ vs. $A G+G G$ ). In both situations, OR values were $<1$, meaning that patients with a G-allelic variant present minimal risk of developing epilepsy (Table III). The frequency of alleles $A$ and $G$ of this polymorphism were represented in similar proportions in the IGE group and in controls, the same being noted after redistributing the patients based on therapeutic control of seizures (recurrent seizures vs. good therapeutic control). Thus this polymorphism had no statistically significant association in the present study.

The distribution of genotypes and allele frequencies for GABRG2 (rs211037) in the three groups of patients showed significant differences, especially in patients with FS (Figure 2). The most 
frequent genotype in group 1 was CC (51.6\%), while in group 2 it was CT heterozygotes (44.4\%). These results are in contradiction to those reported by Chou et al. [32] in which CT heterozygotes were most frequently identified between epilepsy patients and TT homozygotes in those with FS. Compared to the control group, we observed differences in both cases, with a slightly superior CT frequency in epileptics vs. controls ( $40 \%$ and $37.2 \%)$, and the same frequency of CC homozygotes in cases and controls. When comparing patients with and without FS, we found statistically significant differences, the frequency of CT heterozygous and TT homozygote genotypes being superior to that identified in controls. Comparing CT or TT genotype frequency to that of the CC genotype (reference genotype for GABRG2 Asn196Asn polymorphism), we found no risk associated with the development of epilepsy, again in contradiction to the results of Chou et al. [32] In contrast, recessive model C allele carriers (TT vs. CT + CC genotypes) have been identified as having an about 5.5 times higher risk for developing epilepsy compared to those with a T allele genotype ( $p=0.0009)$.

In 2013, Haerian et al. conducted a comprehensive meta-analysis of 8 studies published between 2002 and 2011 on populations of different origin from Asia, Europe and America assessing the GABRG2 rs211037 genotype distribution in patients with epilepsy, FS and healthy populations [33]. Of these only 3 studies supported the hypothesis that synonymous GABRG2 rs211037 polymorphism is a predictive factor in causing epilepsy or FS in children: an Egyptian study with a positive association for FS and two studies from Taiwan associating the polymorphism with FS and IGE. The Egyptian study conducted by Salam et al. was based on genotyping 100 blood samples from children with FS and 120 samples from healthy children, showing a strong association between allele $C$ in the 588 position in the GABRG2 gene and developing FS (OR $=2.15, p<0.0001)$; the frequency of homozygotes for the wild-type CC allele was significantly higher in those with FS [11]. These results are inconsistent with those reported by Ponnala et al., in a study conducted in India on 127 children with FS and IGE, in which $T$ alleles and TT genotypes were identified as associated with FS [34] - our results are in line with these findings. In addition, the distribution was statistically significant for the allelic variant T, associated with IGE in the study by Ponnala et al. [34] (OR = $2.05,95 \% \mathrm{Cl}: 1.02-4.12 ; p<0.05)$. Although our data did not confirm these results, using a compound model we found an association of allele $C$ in genotypic composition (TT vs. CC + CT) with increased susceptibility to develop IGE. The TT genotype carriers were significantly representative in the group with recurrent FS compared to controls, the same being noted for IGE. Our data showed that GABRG2 588T allele carriers have a 5 times increased risk of developing seizures compared to others, and TT homozygotes and CT heterozygotes have a higher risk of developing recurrent seizures and repeated episodes of FS.

Very few studies have reported comparative analyses of drug-resistant and well-controlled forms of epilepsy. In the study by Ponnala et al. [34] recurrent crises were strongly associated with the TT genotype, a result that was not confirmed by a similar study published by Kumari et al. [35]. However, Chou et al. [32] found an association between GABRG2 (SNP211037) and FS but no association regarding intron position polymorphism of GABRG2 rs211014, a conclusion that is in accordance with our results. Still, Wang et al. found an association between the intronic polymorphism of GABRG 2 and $F S$ in a population in Southern China, where the presence of the A allele was observed with a higher incidence [36]. In another study by Chou et al. [16] the CC genotype was found more frequently in those who developed IGE compared to healthy individuals. These results were also confirmed by Kumari et al., who sought GABRG2 association between polymorphism (rs211037) and the development of epilepsy or recurrent seizures [35].

Allele frequency for the GABRG2 rs211037/ C588T/Asn196Asn polymorphism showed differences in geographic distribution, race and origin of selected populations. An allelic distribution similar to that found in our study was reported in populations of Japanese and Chinese origin, as well as some European populations, with the T allele being more frequently highlighted in those with seizures (either IGE, FS, or both); these results are inconsistent with allele frequencies identified in populations from India, Germany and Nigeria [37-39].

Our study is limited by the relatively small number of patients, which could cause false positive results, especially given the fact that in genetic research, sample size is a determining factor, essential for the study's power to detect causal variants in genetic association found in polygenic, multifactorial diseases. The small sample size is due to the fact that not all caregivers signed the informed consent, and neuroimaging investigations were not widely used. Also, as patients with FS are not usually hospitalized, only a few agreed to return for blood sampling.

Nevertheless, the results show a strong, statistically significant association of the $T$ allele and the TT homozygote genotype of GABRG2 C588T with FS. To our knowledge, this is the first study on a pediatric population from Romania that found an association of GABRG2 gene polymorphism with FS. The results suggest that individuals with the $T$ allele genotype variant have a real risk of recurrent FS, while patients with IGE and a T allele 
variant or CT or TT genotype have a high risk of developing a multidrug-resistant form of epilepsy.

In conclusion, C588T polymorphism of the GABRG2 gene might be a predictive genetic marker in triggering febrile convulsions. GABRG2 rs211037 $\mathrm{TT}$ homozygotes and $\mathrm{T}$ allele variants have an increased risk of developing FS $(p=0.001)$. Recurrent crises and repeated episodes of FS are found more often in those with GABRG2 Asn196Asn TT genotype polymorphism - in these cases, the risk for developing IGE is 45 times higher, and the risk of recurrent FS is about 8 times higher compared to individuals without this polymorphism ( $p=0.002$ and $p=0.04$ respectively). Further studies are needed to explain the association between other unique polymorphisms of the GABRG2 gene and FS, as well as the genotype-phenotype relationship of FS in children.

\section{Acknowledgements}

The authors wish to thank Professor Claudia Bănescu, MD, PhD, from the Department of Genetics of the University of Medicine and Pharmacy Târgu Mureş, for permission to use the laboratory equipment and initiating and supervising molecular techniques. Also, we would like to thank Assistant Professor Septimiu Voidăzan, MD, PhD, from the Department of Epidemiology and Preventive Medicine of the University of Medicine and Pharmacy Târgu Mureş, for his help with statistical analysis.

\section{Conflict of interest}

The authors declare no conflict of interest.

\section{References}

1. Baptista PV. Principles in genetic risk assessment. Ther Clin Risk Manag 2005; 1: 15-20.

2. Al-Asmary SM, Kadasah S, Arfin M, et al. Apolipoprotein $\mathrm{E}$ polymorphism is associated with susceptibility to schizophrenia among Saudis. Arch Med Sci 2015; 11: 869-76.

3. Kreile M, Piekuse L, Rots D, et al. Analysis of possible genetic risk factors contributing to development of childhood acute lymphoblastic leukaemia in the Latvian population. Arch Med Sci 2016; 12: 479-85.

4. Motawi T, Salman T, Shaker O, Abdelhamid A. Association of polymorphism in adiponectin (+45 T/G) and leptin $(-2548 \mathrm{G} / \mathrm{A})$ genes with type 2 diabetes mellitus in male Egyptians. Arch Med Sci 2015; 11: 937-44.

5. Huang Y, Yu S, Wu Z, Tang B. Genetics of hereditary neurological disorders in children. Transl Pediatr 2014; 3: 108-19.

6. Benga I. Tratat elementar de neurologie pediatrică (Vol I) Epilepsia și crizele neepileptice [An elementary treatise on pediatric neurology (Vol. 1) Epilepsy and nonepileptic seizures]. 1st ed. Cluj-Napoca (RO), Iuliu Hațieganu 2003.

7. Carranza RD, Hamiwka L, McMahon JM, et al. De novo SCNA1A mutations in migrating partial seizures of infancy. Neurology 2012; 77: 380-3.
8. Mollah MAH, Dey PR, Tarafdar SA, et al. Zinc in CSF of patients with febrile convulsion. Indian J Pediatr 2002; 69: 859-61.

9. Baulac S, Baulac M. Advances on the genetics of Mendelian idiopathic epilepsies. Clin Lab Med 2010; 30: 911-29.

10. Cossette P, Lachance-Touchette P, Rouleau GA. Mutated GABAA receptor subunits in idiopathic generalized epilepsy. In: Jasper's Basic Mechanisms of the Epilepsies [Internet]. $4^{\text {th }}$ edition. Noebels JL, Avoli M, Rogawski MA, Olsen RW, Delgado-Escueta AV (eds.). Oxford University Press, Bethesda (MD) 2012.

11. Salam S, Rahman H, Karam R. GABRG2 gene polymorphisms in Egyptian children with simple febrile seizures. Indian J Pediatr 2012; 79: 1514-6.

12. Ito M, Ohmori I, Nakahori T, Ouchida M, Ohtsuka Y. Mutation screen of GABRA1, GABRB2 and GABRG2 genes in Japanese patients with absence seizures. Neurosci Lett 2005; 383: 220-4.

13. Mulligan MK, Wang X, Adler AL, Mozhui K, Lu L, Williams RW. Complex control of GABA receptor subunit mRNA expression: variation, covariation, and genetic regulation. PLoS One 2012; 7: e34586.

14. Avoli M, Louvel J, Pumain R, Köhling R. Cellular and molecular mechanisms of epilepsy in the human brain. Prog Neurobiol 2005; 77: 166-200.

15. Macdonald RL, Kang JQ, Gallangher MJ. Mutations in GABA receptor subunits associated with genetic epilepsies. J Physiol 2010; 588: 1861-9.

16. Chou IC, Lee CC, Tsai CH, et al. Association of GABRG2 polymorphisms with idiopathic generalized epilepsy. Pediatr Neurol 2007; 36: 40-4.

17. Lu Y, Wang X. Genes associated with idiopathic epilepsies: a current overview. Neurol Res 2009; 31: 135-43.

18. Bouthour W, Leroy F, Emmanuelli C, et al. A human mutation in GABRG2 associated with epilepsy alters the membrane dynamics of $\mathrm{GABA}_{A}$ receptors. Cereb Cortex 2012; 22: 1542-53.

19. Wallace RH, Marini C, Petrou S, et al. Mutant GABA $A_{A}$ receptor gamma2-subunit in childhood absence epilepsy and febrile seizures. Nat Genet 2001; 28: 49-52.

20. Baulac S, Huberfeld G, Gourfinkel Al, et al. First genetic evidence of $G_{A B A}$ receptor dysfunction in epilepsy: a mutation in the gamma2-subunit gene. Nat Genet 2001; 28: 46-8.

21. Haerian BS, Baum L, Kwan P, et al. Contribution of GABRG2 polymorphisms to risk of epilepsy and febrile seizure: a multicenter cohort study and meta-analysis. Mol Neurobiol 2016; 53: 5457-67.

22. Berg AT, Berkovic SF, Brodie MJ, et al. Revised terminology and concepts for organization of seizures and epilepsies: report of the ILAE Commission on Classification and Terminology, 2005-2009. Epilepsia 2010; 51: 676-85.

23. Măruşteri $M$, Bacârea V. Comparing groups for statistical differences: how to choose the right statistical test? Biochem Med 2010; 20: 15-32.

24. Berkovic SF, Harkin L, McMahon JM, et al. De-novo mutations of the sodium channel gene SCN1A in alleged vaccine encephalopathy: a retrospective study. Lancet Neurol 2006; 5: 488-92.

25. American Academy of Pediatrics, Provisional Committee on Quality Improvement and Subcommittee on Febrile Seizures. Clinical practice guideline - febrile seizures: guideline for the neurodiagnostic evaluation of the child with a simple febrile seizure. Pediatrics 2011; 127-389.

26. Cross HJ. Fever and fever related epilepsies. Epilepsia 2012, 53 (Suppl. 4): 3-8. 
27. Jorde LB, Wooding SP. Genetic variation, classification and race. Nature Genet 2004; 36 (11 Suppl.): S28-33.

28. Campbell MC, Tishkoff SA. African genetic diversity: implications for human demographic history, modern human origins, and complex disease mapping. Annu Rev Genomics Hum Genet 2008; 9: 403-33.

29. Sabeti PC, Schaffner SF, Fry B, et al. Positive natural selection in the human lineage. Science 2006; 16: 1614-20.

30. Tsai FJ, Hsieh YY, Chang CC, et al. Polymorphisms for interleukin 1 exon 5 and interleukin 1 receptor antagonist in Taiwanese children with febrile convulsions. Arch Pediatr Adolesc Med 2002; 156: 545-8.

31. Audenaert D, Schwartz E, Claeys KG, et al. A novel GABRG2 mutation associated with febrile seizures. Neurology 2006; 67: 687-90.

32. Chou CH, Ping CT, Huang CC, et al. Association analysis of gamma_2 subunit of-gamma aminobutyric acid type A receptor polymorphisms with febrile seizures. Pediatr Res 2003; 54: 26-9.

33. Haerian BS, Baum L. GABRG2 rs211037 polymorphism and epilepsy: a systematic review and meta-analysis. Seizure 2013; 22: 53-8.

34. Ponnala S, Chaudhari JR, Jaleel MA, et al. Role of MDR1 C3435T and GABRG2 C588T gene polymorphisms in seizure occurrence and MDR1 effect on anti-epileptic drug (phenytoin) absorption. Genet Test Mol Biomarkers 2012; 16: 550-7.

35. Kumari RA, Lakhan RA, Kalita JB, et al. Association of alpha subunit of GABAA receptor subtype gene polymorphisms with epilepsy susceptibility and drug resistance in north Indian population. Seizure 2010; 19: 237-41.

36. Wang X, Xu M, Du L. Association analysis of gamma2 subunit of gamma-aminobutyric acid (GABA) type A receptor and voltage-gated sodium channel type II alpha-polypeptide gene mutation in Southern Chinese children with febrile seizures. J Child Neurol 2007; 22 714-9.

37. Hapmap (2003). Available at www.hapmap.org/hapmappopulations.html. Accessed October 2015.

38. Kananura C, Haug K, Sander T. A splice-site mutation in GABRG2 associated with childhood absence epilepsy and febrile convulsions. Arch Neurol 2002; 59: 1137-41.

39. Gitaí LL, de Almeida DH, Born JP, et al. Lack of association between rs211037 of GABRG2 gene and juvenile myoclonic epilepsy in Brazilian population. Neurol India 2012; 60: 585-8. 